\title{
Mediastinite descendente necrosante pós-angina de Ludwig*
}

\author{
MARICÉlia BROMMELSTROET ${ }^{1}$, JosÉ FIORAVANTE TOSATTI DA ROSA ${ }^{2}$, \\ PAulo CÉsar Buffara Boscardim ${ }^{3}$, CARlos Augusto SCHMIDLIN ${ }^{1}$, SÉrgio SHibata ${ }^{1}$
}

A angina de Ludwig é uma infecção do espaço submandibular originada, em geral, da infecção do $2^{\circ}$ ou $3^{\circ}$ molar inferior. Como conseqüência, pode causar mediastinite descendente necrosante, que representa uma forma grave e rara de infecção mediastinal, a qual exige diagnóstico precoce e tratamento cirúrgico para reduzir a alta mortalidade associada a esta doença. Dois casos de mediastinite descendente necrosante pós-angina de Ludwig foram tratados com excelentes resultados em nosso hospital. A drenagem mediastinal transcervical está justificada em pacientes com doença limitada ao mediastino superior. Porém, sepse com comprometimento extenso do mediastino requer drenagem através de toracotomia sem demora. (J Pneumol 2001;27(5):269-271)

\section{Necrotizing descending mediastinitis afetr Ludwig angina}

Ludwig's angina is an infection of the submandibular space generally caused by an infection of the $2^{\text {nd }}$ or $3^{\text {rd }}$ lower molar. As a consequence, descending necrotizing mediastinitis, a rare and severe form of mediastinal infection, may occur. The descending necrotizing mediastinitis represents a rare form of mediastinal infection. It presents a high mortality and to decrease that rate it is necessary prompt diagnosis and surgical treatment. Two cases of descending necrotizing mediastinitis due to Ludwig's angina were treated with excellent results in our hospital. The transcervical mediastinal drainage is justified in patients with disease limited to the upper mediastinum. Even so, when there is extensive involvement of the whole mediastinum it is suitable the accomplishment of a wide thoracotomy.

Descritores - Angina de Ludwig. Mediastinite. Diagnóstico. Tratamento. Cirurgia.

Key words - Ludwig's angina. Mediastinitis. Diagnosis. Treatment. Surgery.

\section{INTRODUÇãO}

A mediastinite ocorre freqüentemente por perfuração esofágica ou infecção do esterno pós-cirurgia cardíaca. Embora rara, pode originar-se após angina de Ludwig,

\footnotetext{
* Trabalho realizado na Disciplina de Cirurgia Torácica e Cardiovascular do Hospital de Clínicas da Universidade Federal do Paraná, Curitiba, PR

1. Médico Pós-Graduando.

2. Professor Adjunto.

3. Médico Assistente.
}

Endereço para correspondência - Maricélia Brommelstroet, Rua Lourenço Darif, 22 - 82110-140 - Curitiba, PR. Tel. (41) 338-1114; Email: brommelstroet@uol.com.br

Recebido para publicação em 24/11/00. Aprovado, após revisão, em 1/5/01.
Siglas e abreviaturas utilizadas neste trabalho

MDN - Mediastinite descendente necrosante

UTI - Unidade de terapia intensiva

TC - Tomografia computadorizada

PO - Pós-operatório

DI - Dia de internamento

resultando em um quadro clínico grave descrito como mediastinite descendente necrosante (MDN). A demora no diagnóstico e a drenagem imprópria são as principais causas para a alta mortalidade nessa condição ameaçadora, que requer diagnóstico e tratamento precoces. A maior série da literatura apresentou, em 34 anos, somente 43 casos de $\operatorname{MDN}^{(1)}$.

O objetivo deste trabalho é apresentar dois casos de MDN pós-angina de Ludwig, revisando os aspectos importantes do diagnóstico e do tratamento cirúrgico.

\section{DESCRIÇÃO DOS CASOS}

Caso 1: Homem branco de 56 anos, com odontalgia molar inferior esquerda, odinofagia, aumento de volume da região submandibular, dor torácica anterior ventilató- 
rio-dependente. Ao exame físico, apresentava-se febril $\left(38,5^{\circ} \mathrm{C}\right)$, séptico, com aumento de volume submandibular e região parotídea esquerda, abaulamento do palato mole à esquerda e trismo. À ausculta pulmonar apresentava roncos e sibilos difusos. Algumas horas após o internamento houve piora do estado geral, sendo submetido à traqueostomia, drenagem do abscesso cervical e transferido à UTI. No momento da sua internação, seu hemograma apresentava leucocitose com desvio acentuado à esquerda. Os germes isolados na cultura da secreção foram o Streptococcus viridans e o Peptostresptococcus. Na tomografia computadorizada (TC) cervical observou-se coleção residual, sendo realizada nova drenagem cirúrgica. No transoperatório observou-se a presença de uma fístula traqueoesofágica. A TC de tórax mostrou derrame pleural bilateral com colapso dos lobos inferiores, coleção no mediastino superior até altura da carina e no mediastino médio. O paciente foi submetido à toracotomia pósterolateral direita, com ampla abertura da pleura mediastinal. No mesmo tempo cirúrgico foi realizada uma jejunostomia. O paciente retornou à UTI estável e sem drogas vasoativas. A partir do $3^{\circ}$ dia pós-operatório (PO), permaneceu afebril, com melhora importante do quadro séptico e dos exames laboratoriais. No $8^{\circ}$ dia PO, foram retirados os drenos torácicos, após realizar TC de tórax de controle, não mostrando coleções residuais. No $12^{\circ}$ dia PO recebeu alta da UTI e no $17^{\circ}$ dia PO teve alta hospitalar em bom estado geral e local.

Caso 2: Homem branco de 29 anos, tabagista, com odontalgia inferior direita, com aumento de volume local, odinofagia, febre $\left(42^{\circ}\right)$, trismo e disfagia. Ao exame físico, encontrava-se febril $\left(39^{\circ} \mathrm{C}\right)$ e com abscesso submandibular à direita. No momento da sua internação, seu hemograma apresentava leucocitose com desvio à esquerda. Realizadas traqueostomia e drenagem do abscesso submandibular. Os germes isolados na cultura da secreção foram Streptococcus viridans e Bacteroides fragilis. As hemoculturas foram negativas. No $6^{\circ}$ dia de internamento (DI), o paciente continuava febril $\left(39^{\circ} \mathrm{C}\right)$ e com piora do estado geral; transferido à UTI. Realizada nova drenagem cirúrgica do abscesso. As TC cervical e torácica mostraram aumento de volume da região cervical, presença de coleção hipodensa na região submandibular e supraclavicular direita. Espessamentos pleurais posteriores e opacidades pulmonares maldelimitadas nas bases. Devido à persistência do quadro infeccioso e dos achados da TC, o paciente foi submetido à toracotomia póstero-lateral direita com ampla abertura da pleura mediastinal. No pósoperatório imediato não houve necessidade de drogas vasoativas e permaneceu afebril. No 6을 dia PO, após TC de tórax de controle, foram retirados os drenos. Recebeu alta da UTI no 8으 dia PO e alta hospitalar no 15으 dia PO em bom estado geral e local.

\section{COMENTÁRIOS}

A mais terrível e provavelmente a forma mais letal de mediastinite é a variedade descendente necrosante que ocorre como complicação de infecção da orofaringe, sendo uma entidade clínica incomum. As infecções dos espaços cervicais profundos passaram a ser quadros mais infreqüentes desde a introdução dos antibióticos na prática clínica, na década de 1940. Embora rara, esta variedade de mediastinite é uma doença altamente letal, com taxa de $40 \%$ de mortalidade na era antibiótica ${ }^{(2-4)}$. Acomete, normalmente, pacientes jovens, a maioria do sexo masculino, e existe associação com etilismo crônico(5).

A infecção origina-se nos planos das fáscias da cabeça e do pescoço, infiltra-se para o mediastino, ao longo das fáscias cervicais, facilitada pela gravidade, respiração $e$ pressão intratorácica negativa. $\mathrm{O}$ trajeto anatômico mais comum da propagação da infecção é pelo espaço faríngico lateral do espaço retrovisceral (retrofaríngico), estendendo-se inferiormente ao mediastino; isso ocorre em $70 \%$ dos $\operatorname{casos}^{(4,6)}$. A causa mais comum de infecção cervical profunda na infância é a amidalite aguda, que pode levar ao abscesso peritonsilar e, nos adultos, os focos dentários podem provocar abscessos no assoalho da boca ${ }^{(7)}$.

A angina de Ludwig é um quadro clássico, descrito em 1836 por Wilhelm von Ludwig, e consiste na infecção do espaço submandibular (espaço sublingual e espaço submaxilar). Para afirmar-se o diagnóstico de angina de Ludwig, as seguintes características devem ser observadas: o processo inicia-se no assoalho da boca, usualmente com a infecção do $2^{\circ}$ ou $3^{\circ}$ molar inferior; a disseminação ocorre para o espaço submandibular, mais por contigüidade, pelos planos das fáscias, do que pelos vasos linfáticos; a infecção apresenta-se como endurecimento da região submandibular, sem formação de muita secreção purulenta; o processo poupa as glândulas salivares e linfonodos; a infecção é geralmente bilateral.

O diagnóstico de infecção cervical é clinicamente óbvio, mas o diagnóstico precoce de mediastinite é freqüentemente difícil pela incerteza dos sintomas iniciais ${ }^{(8)}$. Devese suspeitar de mediastinite quando há a ocorrência de sintomas respiratórios como dor torácica, dispnéia ou angústia respiratória associados a abscesso cervical ${ }^{(1)}$.

As radiografias do pescoço e do tórax podem revelar várias características; entretanto, esses sinais aparecem tardiamente no curso da doença e, além disso, procedimentos prévios (drenagem cervical, traqueostomia e acesso venoso central) tornam difícil a interpretação das radiografias de tórax. A TC é extremamente útil nas infecções cervicais, porque mostra com exatidão o envolvimento precoce do mediastino, permitindo a localização ideal à intervenção cirúrgica ${ }^{(4)}$. Os critérios de Estrera et al. também são utilizados para o diagnóstico da $\mathrm{MDN}^{(2)}$. 
O emprego isolado de antibioticoterapia intravenosa de amplo espectro não é eficiente sem a drenagem cervical adequada, desbridamento extenso e excisão do tecido necrótico com irrigação mediastino-pleural ${ }^{(1)}$. Na maioria dos casos, repetidas drenagens cervicais podem ser necessárias, devido à presença de infecção persistente.

Para muitos autores, o acesso cirúrgico ideal para drenagem do mediastino, em pacientes com MDN, é dependente do nível de difusão do processo necrótico ${ }^{(1,2,9-11)}$. Se a infecção alcança só o mediastino superior, acima do nível da carina, a drenagem mediastinal transcervical pode ser adequada. A mediastinotomia anterior ou a drenagem subxifóide, associada à drenagem cervical, também podem ser realizadas em pacientes extremamente instáveis hemodinamicamente, apesar da existência de mediastinite extensa, até a melhora parcial do quadro ${ }^{(4)}$. No caso de mediastinite que vai além da bifurcação da traquéia ou ao nível da quarta vértebra torácica, é recomendada a drenagem do mediastino por toracotomia póstero-lateral, que proporciona exposição adequada da cavidade pleural, do pericárdio e de todos os compartimentos do mediastino ${ }^{(2,8)}$. Ris et al. relataram sucesso no tratamento de dois em três pacientes com MDN que tiveram drenagem mediastinal pelo acesso de Clamshell, o qual inclui toracotomia anterior bilateral com esternotomia transversa ${ }^{(12)}$. As vantagens com esse acesso são: cirurgia em um único tempo, excelente exposição para descorticação bilateral, desbridamento do mediastino e pericardiectomia. Porém, essa cirurgia é particularmente invasiva nos pacientes extremamente graves, expondo-os ao risco de paralisia do nervo frênico, osteomielite do esterno, deiscência de sutura do esterno e risco de óbito mais elevado. A esternotomia mediana também parece inadequa-

\section{REFERÊNCIAS}

1. Marty-Ané $\mathrm{CH}$, Alauzen M, Alric P, Cousine OS, Mary H. Descendig necrotizing mediastinitis. J Thorac Cardiovasc Surg 1994;107:55-61.

2. Estrera AS, Lanay MJ, Grisham JM. Descending necrotizing mediastinitis. Surg Gynecol Obstet 1983;157:545-552.

3. Levine TM, Wurster CF, Krepsi YP. Mediastinitis occurring as a complication of odontogenic infections. Laryngoscopy 1996;747:1982-1984.

4. Wheatley MJ, Stirling MC, Kirsh MM, Gago O, Orringer MB. Descending necrotizing mediastinitis: transcervical drainage is not enough. Ann Thorac Surg 1990;49:780-784.

5. Cordero L, Torre W, Freire D. Descending necrotizing mediastinitis and respiratory distress syndrome treated by aggressive surgical treatment. J Cardiovasc Surg 1996;37:87-88.

6. Takao M, Ido M, Hamaguchi K, Chikusa H, Namikawa S, Kusagawa $\mathrm{M}$. Descending necrotizing mediastinitis secondary to a retropharyngeal abscess. Eur Respir J 1994;7:1716-1718.

7. Durazzo MD, Pinto FR, Rocha MSL, Volpi EM, Nishio S, Brandão LG, Ferraz AR, et al. Os espaços cervicais profundos e seu interesse nas infecções da região. Rev Assoc Med Bras 1997;43:119-126.

8. Marty-Ané $\mathrm{CH}$, Berthet JP, Alric P, Pegis JD, Rouvière P, Mary $\mathrm{H}$. Management of descending necrotizing mediastinitis: an aggressive treatment for an aggressive disease. Ann Thorac Surg 1999;68:212-217. da em MDN, pela incidência de osteomielite, deiscência de sutura do esterno e ser o acesso para o compartimento póstero-basal da cavidade torácica inadequado, especialmente no lado esquerdo ${ }^{(4)}$.

Roberts et al. relataram a realização de toracoscopia em um caso de MDN com abscesso mediastinal encapsulado e Gobien et al. propuseram drenagem percutânea guiada por TC como uma valiosa alternativa para intervenção cirúrgica em pacientes selecionados ${ }^{(13,14)}$. Esses abscessos normalmente são decorrentes de perfuração esofágica, de complicação pós-operatória, ou de hematoma mediastinal pós-trauma infectado e nesses casos a toracoscopia pode ser útil ou até mesmo a drenagem percutânea guiada por $\mathrm{TC}^{(15)}$. Em contraste, a MDN é uma infecção difusa que envolve o pescoço e o mediastino, sendo imprópria a toracoscopia ou a drenagem percutânea, porque a presença de coleção fluida, não encapsula$\mathrm{da}$, associada com celulite necrosante extensa, requer desbridamento cirúrgico.

A ocorrência de erosão dos vasos cervicais com sangramento abundante não é uma complicação infreqüente de infecções profundas do pescoço. A presença de dor abdominal em paciente com MDN deve ser seriamente considerada, porque pode ser uma indicação de extensão retroperitoneal. Esses pacientes estão mais propensos a ter outras complicações como broncoaspiração, paralisia dos pares cranianos, abscessos cerebrais, fasciite necrosante, síndrome da angústia respiratória do adulto e falência de múltiplos órgãos ${ }^{(5,11,16)}$.

A traqueostomia é considerada, por alguns autores, como parte integrante dos cuidados nos casos de infecções cervicofasciais graves, sendo necessária em $50 \%$ dos casos e alguns a realizam profilaticamente $e^{(4,7)}$.

9. Haraden BM, Zwemer FL. Descending necrotizing mediastinitis: complication of a simple dental infection. Ann Emerg Med 1997;5:683686.

10. Brunelli A, Sabbatini A, Catalini G, Fianchini A. Descending necrotizing mediastinitis: cervicotomy or thoracotomy. J Thorac Cardiovasc Surg 1996;111:485-486.

11. Temes RT, Crowell RE, Mapel DW, Ketai L, Pett SB, Wernly A. Mediastinitis without antecedent surgery. Thorac Cardiovasc Surg 1998; 46:84-88.

12. Ris HB, Banic A, Furrer M. Descending necrotizing mediastinitis. Ann Thorac Surg 1996;62:1650-1654.

13. Roberts JR, Smythe R, Weber RW. Thoracoscopic management of descending necrotizing mediastinitis. Chest 1997;112:850-854.

14. Gobien RP, Stanley JH, Gobien BS, Vujic I, Pass HI. Percutaneous catheter aspiration and drainage of suspected mediastinal abscesses. Radiology 1984;151:69-71.

15. Laisaar T. Video-assisted thoracoscopic surgery in the management of acute purulent mediastinitis and pleural empyema. Thorac Cardiovasc Surg 1998;46:51-54.

16. Alexander DW, Leonard JR, Trail ML. Vascular complications of deep neck abscesses. Laryngoscope 1968;78:361. 